\title{
shutdown is a component of the Drosophila piRNA biogenesis machinery
}

\author{
JONATHAN B. PREALL, ${ }^{1}$ BENJAMIN CZECH, ${ }^{1}$ PALOMA M. GUZZARDO, FELIX MUERDTER, \\ and GREGORY J. HANNON ${ }^{2}$ \\ Howard Hughes Medical Institute, Watson School of Biological Sciences, Cold Spring Harbor Laboratory, \\ Cold Spring Harbor, New York 11724, USA
}

\begin{abstract}
In animals, the piRNA pathway preserves the integrity of gametic genomes, guarding them against the activity of mobile genetic elements. This innate immune mechanism relies on distinct genomic loci, termed piRNA clusters, to provide a molecular definition of transposons, enabling their discrimination from genes. piRNA clusters give rise to long, single-stranded precursors, which are processed into primary piRNAs through an unknown mechanism. These can engage in an adaptive amplification loop, the ping-pong cycle, to optimize the content of small RNA populations via the generation of secondary piRNAs. Many proteins have been ascribed functions in either primary biogenesis or the ping-pong cycle, though for the most part the molecular functions of proteins implicated in these pathways remain obscure. Here, we link shutdown (shu), a gene previously shown to be required for fertility in Drosophila, to the piRNA pathway. Analysis of knockdown phenotypes in both the germline and somatic compartments of the ovary demonstrate important roles for shutdown in both primary biogenesis and the ping-pong cycle. shutdown is a member of the FKBP family of immunophilins. Shu contains domains implicated in peptidyl-prolyl cis-trans isomerase activity and in the binding of HSP90-family chaperones, though the relevance of these domains to piRNA biogenesis is unknown.
\end{abstract}

Keywords: piRNAs; transposon silencing; RNAi; FKBP; germ cells

\section{INTRODUCTION}

Eukaryotic genomes are prone to the accumulation of repetitive sequences, including transposable elements, over evolutionary time (McClintock 1953; Kim et al. 1994; Brennecke et al. 2007; Chambeyron et al. 2008; Feschotte 2008). The genomic instability brought about by transposon activity is a double-edged sword. Low levels of transposition can drive evolution in the long term, but loss of control over mobile elements in any individual can threaten reproductive success. Mechanisms for suppressing transposon activation in the germline are therefore both potent and widely conserved (Grimson et al. 2008). In animals, the PIWI-interacting RNA (piRNA) pathway is key to transposon silencing in reproductive tissues (Aravin et al. 2006; Girard et al. 2006; Lau et al. 2006; Vagin et al. 2006; Malone and Hannon 2009; Khurana and Theurkauf 2010; Senti and Brennecke 2010). In Drosophila, piRNAs are active both in the germ cell lineage

\footnotetext{
${ }^{1}$ These authors contributed equally to this work.

${ }^{2}$ Corresponding author

E-mail hannon@cshl.edu

Article published online ahead of print. Article and publication date are at http://www.rnajournal.org/cgi/doi/10.1261/rna.034405.112.
}

and in a particular somatic lineage that encysts the germ cells and provides growth and maturation signals (Malone et al. 2009).

piRNA clusters sit at the apex of the pathway and, based upon their sequence content, define transposon targets for repression (Brennecke et al. 2007). piRNA clusters give rise to long, single-stranded transcripts (Brennecke et al. 2007) that are thought to be exported to the cytoplasm and processed into primary piRNAs, most likely in specialized cytoplasmic structures (Saito et al. 2010; Handler et al. 2011). A number of proteins have been implicated in primary piRNA biogenesis and their loading into PIWI-family proteins, including Armitage, Zucchini, Vreteno, and the $\mathrm{Yb}$ family (Klattenhoff et al. 2007; Pane et al. 2007; Malone et al. 2009; Szakmary et al. 2009; Haase et al. 2010; Olivieri et al. 2010; Saito et al. 2010; Handler et al. 2011; Zamparini et al. 2011). Yet, almost nothing is known about how each of these promotes the production of primary piRNAs.

The soma relies on a single piRNA cluster, flamenco (flam) (Brennecke et al. 2007). This $\sim 180 \mathrm{~kb}$, centromereproximal locus on the $\mathrm{X}$ chromosome produces a piRNA population that is strongly enriched for species antisense to the gypsy family elements. These elements are active in follicle cells and can propagate by infection of germ cells through 
their capability to form virus-like particles (Pelisson et al. 1994; Chalvet et al. 1999). Somatic piRNAs are produced solely through primary biogenesis (Brennecke et al. 2007; Malone et al. 2009). In the germline, a greater variety of clusters targets a broad spectrum of mobile elements and engages an adaptive cycle, termed ping-pong, through which transposon mRNAs help to shape piRNA populations (Brennecke et al. 2007; Gunawardane et al. 2007). Here, antisense-oriented piRNAs derived from genomic clusters are loaded into Aubergine (Aub) and cleave active transposable element transcripts in an RNAi-like reaction. Unlike classical RNAi, this triggers the production of a new small RNA, derived from the target mRNA and with its $5^{\prime}$ end formed by Aub-mediated cleavage. The new, secondary piRNA is loaded into Ago3, which can then use this sense-oriented species to recognize and cleave cluster-derived transcripts, producing more antisense piRNAs via a similar mechanism.

Mutations in the Drosophila piRNA pathway generally result in sterility with stereotypical phenotypes in the male and female germline (Schupbach and Wieschaus 1991; Wilson et al. 1996; Gonzalez-Reyes et al. 1997; Lin and Spradling 1997; Cox et al. 2000; Cook et al. 2004). In part, these are thought to result from DNA double-strand breaks induced by element activity (Chen et al. 2007; Klattenhoff et al. 2007). Such breaks trigger meiotic checkpoint activation mediated by the Drosophila chk2 ortholog, loki, which in turn disrupts dorsal-ventral axis formation during oogenesis. Hence, mutations in secondary piRNA genes such as aubergine display fused dorsal appendages and other hallmarks of oocyte ventralization (Theurkauf et al. 2006). Transposon silencing is also critical for the maintenance of germline stem cells (Lin and Spradling 1997; Cox et al. 2000; Houwing et al. 2007). In the male germline, loss of Su(ste) piRNAs derepresses the repetitive Stellate locus, which disrupts spermiogenesis by causing the overproduction and eventual crystallization of Stellate protein within the testis (Bozzetti et al. 1995; Aravin et al. 2001). Several mutants that are now known to affect the Drosophila piRNA pathwayincluding aubergine, zucchini, squash, vasa, and cutoff-were first described in a female sterility screen by Schüpbach and Wieschaus over $20 \mathrm{yr}$ ago (Schupbach and Wieschaus 1989, 1991). Of the genes identified in that study that would eventually come to be known as piRNA factors, all but cutoff were classified phenotypically as having defects in dorsal appendage formation (Schupbach and Wieschaus 1991).

Subsequently, Munn and Steward (2000) mapped another of these female sterile mutations, shutdown (shu, CG4735), to an immunophilin gene of the FK506-binding protein (FKBP) family. Mutations in shu disrupt germ cell division, eventually causing the germline stem cells to fail entirely. Two strong alleles caused sterility in both males and females, while a third point mutant allele did not affect male fertility. In mutant females, stem cells that successfully divide generally produce faulty egg chambers that arrest mid-oogenesis. Germline clones for strong alleles of shu can produce mature oocytes, though they display typical patterning defects such as fused dorsal appendages. Considered together, these observations implicate $s h u$ as a component of the Drosophila piRNA pathway. This conjecture is supported by the presence of FKBP6, the mammalian protein most similar to Shutdown, in complexes with mammalian Piwi-family proteins, Miwi and Miwi2 (Vagin et al. 2009).

FKBPs play diverse biological roles ranging from facilitating protein folding to modulating transport (Ahearn et al. 2011), receptor signaling (Li et al. 2011), and meiotic recombination (Crackower et al. 2003; Kang et al. 2008). The FKBP domain is annotated as a peptidyl-prolyl cistrans isomerase (PPIase), though there are many instances of well-conserved FKBP domains that lack PPIase activity (Gollan and Bhave 2010). The macrolide immunosuppressants FK506 (tacrolimus) and rapamycin (sirolimus) bind with sub-nanomolar affinity to the FKBP domain and block a key protein-protein interaction surface, but as is the case with PPIase activity, many family members display much reduced affinities for these compounds (DeCenzo et al. 1996; Gollan and Bhave 2010).

FKBP-class immunophilins display a variety of domain architectures. One arrangement, conserved from protozoa to humans, places a tetratricopeptide repeat (TPR) domain downstream from the FKBP domain (Pratt et al. 2004). The TPR domain is a protein-protein interaction module that binds heat shock proteins (HSPs), primarily of the HSP90 family in higher eukaryotes (Pratt 1998; Allan and Ratajczak 2011). Several crystal structures are available that highlight key conserved residues that participate in this interaction (Van Duyne et al. 1993; Ward et al. 2002). Connections between small RNA silencing pathways and HSP activity have been observed in several model systems (Smith et al. 2009). In particular, RNA-induced silencing complex (RISC) loading is facilitated by HSP90 and ATP hydrolysis (Iki et al. 2010; Iwasaki et al. 2010; Miyoshi et al. 2010; Iki et al. 2011).

Here, we report that shutdown is a critical element of the Drosophila piRNA pathway. Tissue-specific depletion of Shu results in derepression of transposon expression and a near-complete loss of mature piRNAs in both the somatic and germline lineages. Shu is cytoplasmically localized, and its loss disrupts the localization of all three piRNA effectors, Piwi, Aub, and Ago3. We hypothesize that Shu is an essential component of both primary and ping-pongderived piRNA biogenesis, likely acting at a very early step that is shared between both piRNA systems.

\section{RESULTS AND DISCUSSION}

\section{Clues to a role for FKBPs in the piRNA pathway}

We previously carried out a proteomic analysis of mammalian PIWI proteins, Miwi and Miwi2 (Vagin et al. 2009). Among the components of these complexes were murine 
FKBP6 and multiple HSPs. FKBP5 was also detected in Miwi immunoprecipitates with roughly half the coverage seen for FKBP6. Given the greater convenience of manipulating the piRNA pathway in Drosophila, we chose to examine potential roles for FKBP proteins in that model system.
The Drosophila genome encodes eight FKBP family members (Fig. 1A,B). Three, CG1847, CG5482, and FKBP59, are annotated to share the domain architecture of FKBP6, with their FKBP domains followed by a TPR. Shutdown is a potential fourth member of this group. Though its TPR

A

\begin{tabular}{|c|c|}
\hline \multicolumn{2}{|c|}{ FKBP_C : Active Site } \\
\hline Dm Shu & 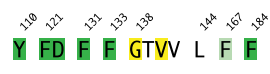 \\
\hline $\begin{array}{l}\text { Hs } \\
\text { FKBP12 } \\
\text { Dm FK506-bp2 } \\
\text { Dm FK506-bp1 } \\
\text { Dm FKBP13 } \\
\text { Dm CG14715 } \\
\text { Dm FKBP59 } \\
\text { Dm CG5482 } \\
\text { Dm CG1847 } \\
\text { At FKBP42 }\end{array}$ & 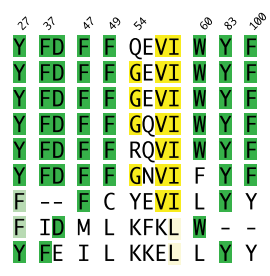 \\
\hline
\end{tabular}

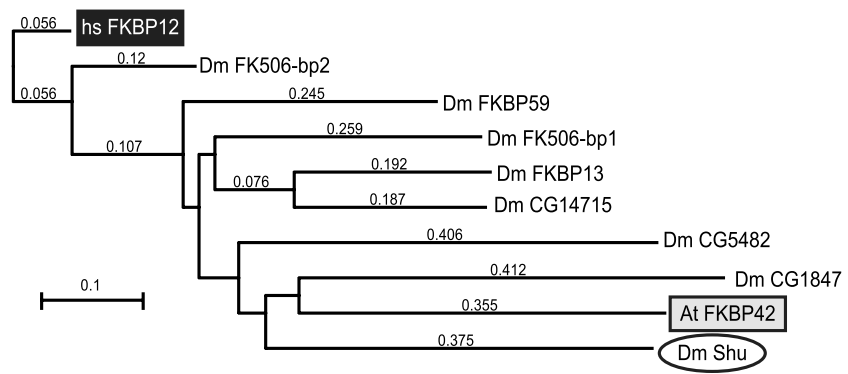

\section{TPR : HSP90-interacting}
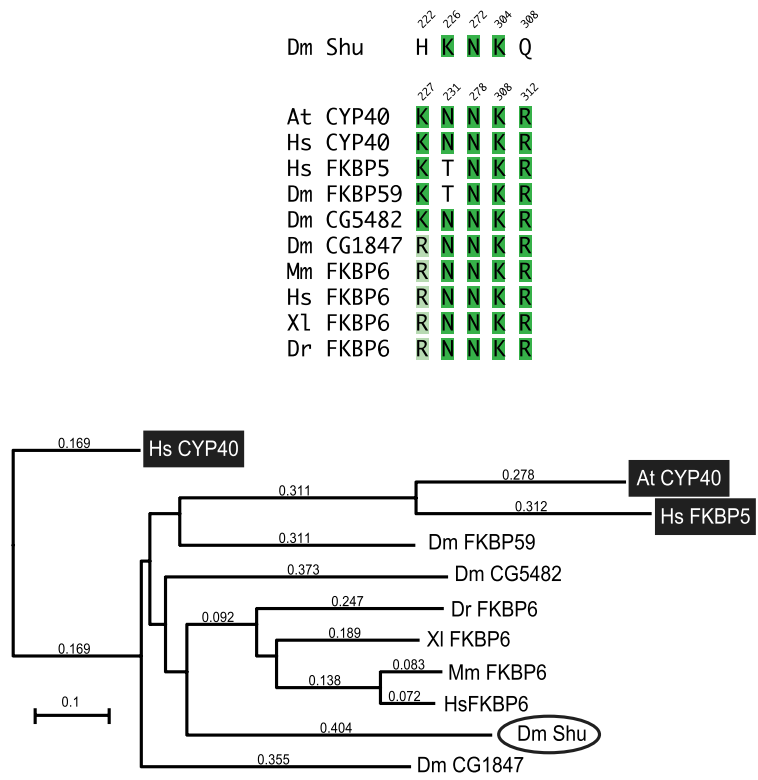

\section{B}

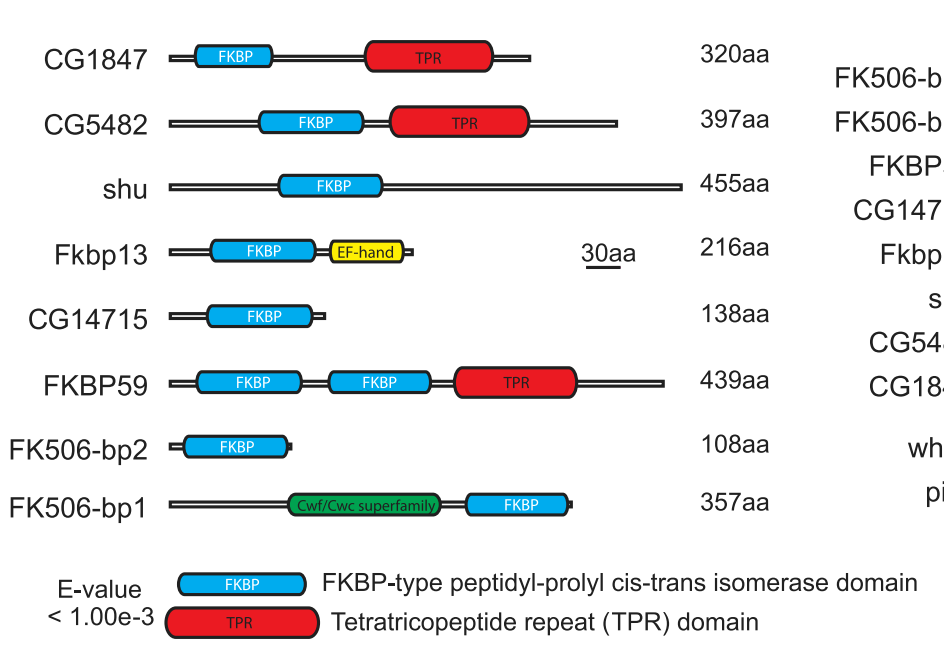

C

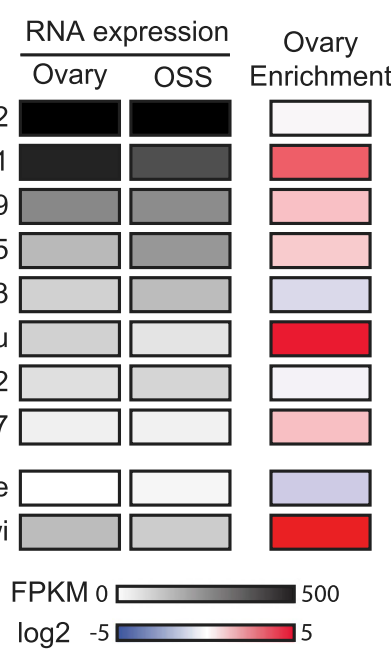

D

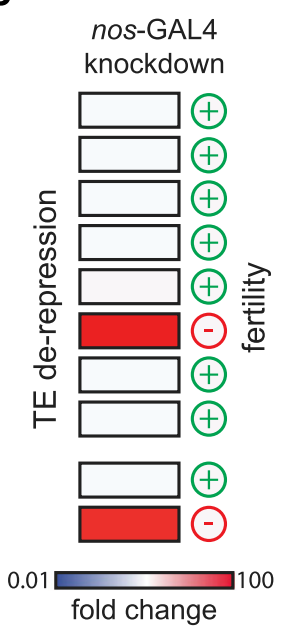

FIGURE 1. Shutdown is the only FKBP-family protein required for transposon silencing. (A) Above are shown the critical residues for the FKBP family peptidyl-prolyl cis-trans isomerase active site and the HSP90-interacting region in the TPR, as indicated, comparing the site in Drosophila Shutdown with those present in other family members. Residues in green indicate highly conserved residues with a known impact on PPIase activity, while those in yellow indicate a more poorly conserved region that has also been implicated. Below are evolutionary trees comparing each domain with family members present in other species. (B) The domain structures of the eight Drosophila FKBP family members are shown schematically. $(C)$ Relative expression levels of Drosophila FKBP family members are shown for ovary and OSS RNAseq data sets. Relative enrichment in ovary versus other tissues is also shown. (D) Shown are relative HetA expression levels detected in ovaries from Drosophila engineered to express dsRNAs corresponding to each family member in the germline lineage. To the right is indicated whether dsRNA-expressing females are fertile $(+)$ or sterile $(-)$. 
domain has substantially diverged in comparison to its paralogs (TPR_2, Pfam e-value $=0.0026)$, secondary structure predictions using the Phyre2 algorithm (www.sbg.bio.ic. ac.uk/phyre2) annotate the putative TPR as such with high confidence. Among Drosophila FKBPs, Shutdown is most closely related to FKBP6 $\left(\mathrm{E}=2 \times 10^{-36}\right)$ overall, whereas FKBP59 is a potential FKBP5 ortholog $\left(\mathrm{E}=2 \times 10^{-46}\right)$.

An examination of RNAseq both from the Drosophila ovarian somatic sheet (OSS) cell line and from a published ovarian data set revealed that several FKBPs are expressed in female reproductive tissues (Fig. 1C; Gan et al. 2010). A broader set of published microarray data (Chintapalli et al. 2007) suggested that the expression pattern of shu is much more biased to the ovary than is expression of other family members (Fig. 1C), a bias shared by many piRNA pathway components.

The FKBP_C domain is broadly conserved across evolution, though its PPIase activity is not (Kamphausen et al. 2002). Phylogenetic comparison of Drosophila FKBP_C domains to known active (Homo sapiens FKBP12) and inactive (Arabidopsis thaliana FKBP42) PPIase domains suggested that shu is more similar to inactive variants (Fig. 1A, bottom). Shutdown does retain more of the active site residues shown to be essential for PPIase activity in human FKBP12 (Fig. 1A, left) than does AtFKBP42. A conserved tryptophan residue (W60 in $H s F k b p 12$ ) has been replaced by a leucine in Shutdown. Introduction of this change into Fkbp12 reduces PPIase activity by approximately eightfold and rapamycin and FK506 binding affinity by 10- and 75fold, respectively. It is therefore likely that Shutdown does not represent an optimally active PPIase and may instead utilize the domain as a protein interaction interface, as do other FKBP family members (Gollan and Bhave 2010).

The Shu TPR domain is less well conserved and, in fact, shows little similarity to other TPRs known to bind HSP90 (Fig. 1A, right). In particular, nonconservative amino acid changes at two key residues suggested that the affinity of this domain for the C-terminal MEEVD of HSP90 is likely to be dramatically reduced compared with other family members (Ratajczak and Carrello 1996; Ward et al. 2002). Still, a shu allele $\left(s h u^{P B 70}\right)$ bearing a point mutation at a nonconserved residue in the putative TPR is sufficient to cause female sterility, indicating that this region is essential for some aspects of Shu function.

\section{Shutdown is implicated in transposon silencing}

Recent work has suggested that Dcr-2 is a limiting factor that prevents conventional dsRNA triggers from inducing potent RNAi in Drosophila germ cells, but that this restriction could be overcome by enforced Dcr-2 expression (Handler et al. 2011; Wang and Elgin 2011). We took advantage of this observation by bringing UAS-driven dsRNA constructs from the Vienna Drosophila RNAi Center (VDRC) into a background containing a germline-specific GAL4- driver (\{GAL4-nos.NGT\}40; aka nos-GAL4) and a UASDcr-2 transgene. Among dsRNAs targeting all fly FKBP proteins, only those corresponding to $s h u$ had significant impacts on expression levels of the HetA transposon (Fig. 1D). Moreover, only dsRNAs targeting Shu caused female sterility, a property typical of piRNA mutants (Fig. 1D).

To validate $s h u$ as a novel piRNA pathway component, we compared the impact of its depletion to knockdowns of known piRNA pathway genes, armi and piwi. Germline silencing of each gene resulted in a similar level of derepression for 17 transposons, measured by quantitative PCR (qPCR) (Fig. 2A). The tissue specificity of our knockdown strategy was supported by the fact that germlinespecific, telomeric transposons TAHRE, HetA, and TART were the most heavily derepressed (greater than 150-fold, $P<0.01$ ), whereas RNA levels for primarily somatic elements, such as ZAM, remained unchanged (about 1.2- to 1.5 -fold).

Shu RNAi also recapitulated the ventralized egg phenotype of $s h u^{P B 70}$ germline clones, as evidenced by a high incidence of fused or abnormal dorsal appendages (Fig. 2B; Munn and Steward 2000). Surprisingly, the ventralization phenotype was not penetrant in armi and piwi knockdowns eggs, despite the eggs being nonviable (Fig. 2C). For armi, prior studies of mutants produce a clear expectation of ventralization upon potent knockdown (Klattenhoff et al. 2007; Orsi et al. 2010). For piwi, the prediction is less clear. Germline piwi clones were reported not to show this distinctive phenotype; however, RNAi-mediated piwi knockdown did produce eggs with a spindle morphology (Cox et al. 2000; Wang and Elgin 2011). In addition to causing sterility, shu depletion also reduced the number of nonviable eggs laid, suggesting that there may be additional requirements for $s h u$ function outside of piRNA-mediated transposon silencing.

\section{Shu is required for Piwi, Aub, and Ago3 localization}

In wild-type tissues, Piwi is localized to the nucleus of germline and somatic cells (Cox et al. 2000; Saito et al. 2006; Brennecke et al. 2007). Aub and Ago3 are expressed exclusively in the germline and are enriched in a perinuclear organelle called nuage (Lim and Kai 2007; Li et al. 2009). Proper localization depends upon normal piRNA production and loading into PIWI family proteins, and disruption of this pattern is an indicator of impaired biogenesis (Malone et al. 2009).

Depletion of $s h u$ using the nos-GAL4 driver resulted in redistribution of Piwi from nurse cell nuclei to the syncytial cytoplasm of the developing egg chamber, while neighboring somatic follicle cells retain proper nuclear Piwi localization (Fig. 2D). Similarly, the ping-pong factors Ago3 and Aub were redistributed from nuage to cytoplasmic foci, while the localization of the core nuage component Vasa was not altered (Fig. 2D). Driving the $s h u$ dsRNA using 
A
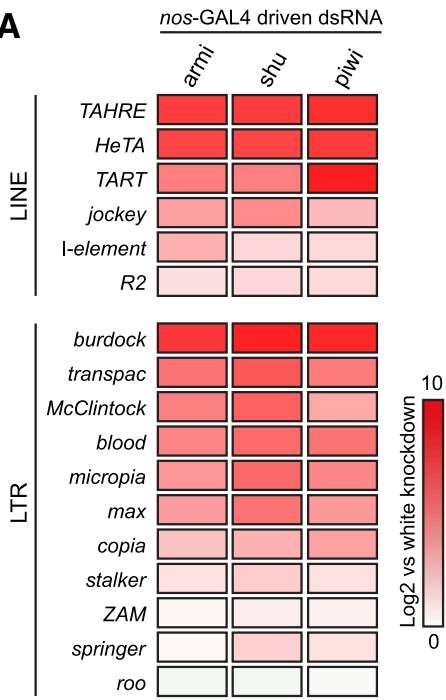

B

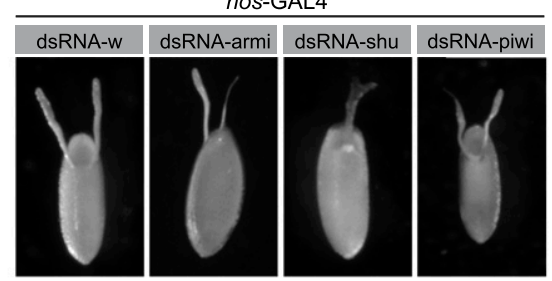

C

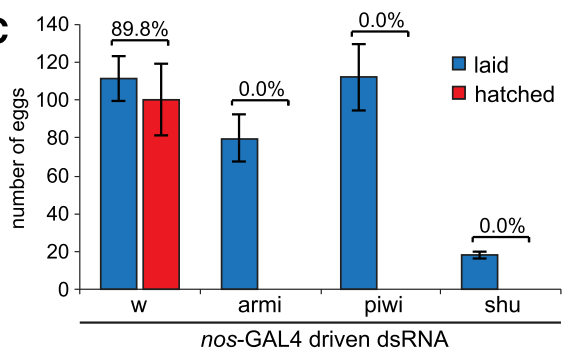

D

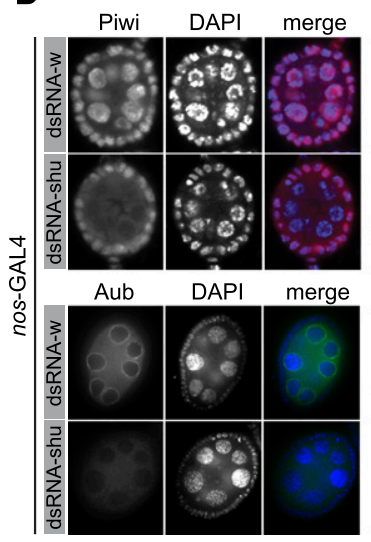

E

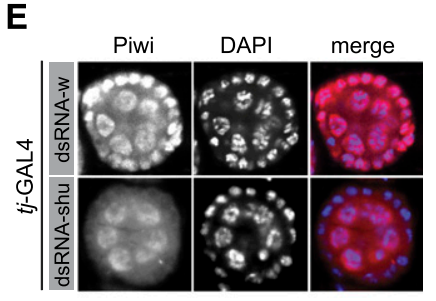

FIGURE 2. Phenotypes of Drosophila with germline-specific shu knockdown. (A) Depletion of $s h u$ in the germline results in derepression of multiple, unrelated transposons from the LINE and LTR families. Derepression, relative to white RNAi, is displayed as $\log 2$ fold change in heat map form. Analysis of flies with germline knockdown of Armi and Piwi, two known piRNA components, is displayed for comparison. (B) Germline-knockdown of shu causes patterning defects as indicated by the presence of fused dorsal appendages. (C) Depletion of shu causes female sterility. shu RNAi females lay fewer eggs compared with controls or animals depleted of other piRNA pathway factors. Hatching rates for all knockdown animals are zero, indicating complete sterility. (D) Depletion of $s h u$ in the germline using nos-GAL4 results in Piwi delocalization from nuclei and in Aub and Ago3 delocalization from nuage. Vasa localization is not changed. Depletion of white is shown as control. (E) Tj-GAL4-driven knockdown of shu in somatic follicle cells also causes Piwi delocalization. RNAi against white is shown as control.

OSS cells. Control constructs showed the expected localization with GFPPiwi accumulating in nuclei and with GFP-Armi showing strong perinuclear localization consistent with its association with Yb-bodies. Zucchini features sequence homology with phospholipase $\mathrm{D}$ and was reported to localize to the outer membrane of mitochondria. In our studies, it displayed considerable overlap with the mitochondrial stain MitoTracker CMXRos (Supplemental Fig. S2). While cytoplasmic foci of GFPtagged Shu were visible using both $\mathrm{N}$ - and C-terminal constructs, they did not overlap with the previously characterized localization patterns of other piRNA pathway proteins. Considered together, these data indicate that Shu is neither enriched in known structures associated with silencing nor required for assembly of a core nuage component.

\section{Shu is essential for accumulation of both primary and secondary piRNAs}

Strong derepression of germline and somatic transposons and the loss of characteristic localization patterns for Piwi-family proteins suggested that $s h u$ might function as a core piRNA biogenesis component, similar to armi. To address this possibility, we cloned and sequenced small RNAs from ovaries in which we drove the expression of white $(w), s h u$, and piwi dsRNAs in the germline (nos-GAL4) or soma ( $t j$-GAL4), as described above. Germline small RNAs libraries were normalized using the number of unique reads mapping to the flam locus, which is unaffected by germlinespecific knockdowns. Germline-specific shu knockdown dramatically reduced the

GAL4 expressed from the soma-specific traffic jam promoter ( $t j$-GAL4) caused delocalization of Piwi from the nuclei of follicle cells, while germline Piwi remained unaffected (Fig. 2E).

Despite its effects on the localization of PIWI-family proteins, we found that the bulk of Shutdown was not associated with domains characteristic of those piRNA pathway components. We generated $\mathrm{N}$ - and C-terminal GFP fusions of Shu expressed under the control of the ubiquitous Actin5c promoter. We examined the localization of Shutdown fusion proteins by transfection of observed piRNA population compared with the white knockdown control. Small RNA reads with the characteristic piRNA size (23-29 nucleotides [nt]) mapping to the germline-specific, dual-strand $42 A B$ cluster were reduced 11.4fold overall $(8.2 \times$ on plus strand, $14.4 \times$ on minus strand). In contrast, piwi knockdown produced only a 2.8 -fold overall reduction $(2.8 \times$ on plus strand, $2.7 \times$ on minus strand) (Fig. 3A).

The incomplete loss of piRNAs in the piwi knockdown likely reflects the fact that piRNAs from $42 A B$ are normally loaded into each of the three Drosophila PIWI proteins 
A



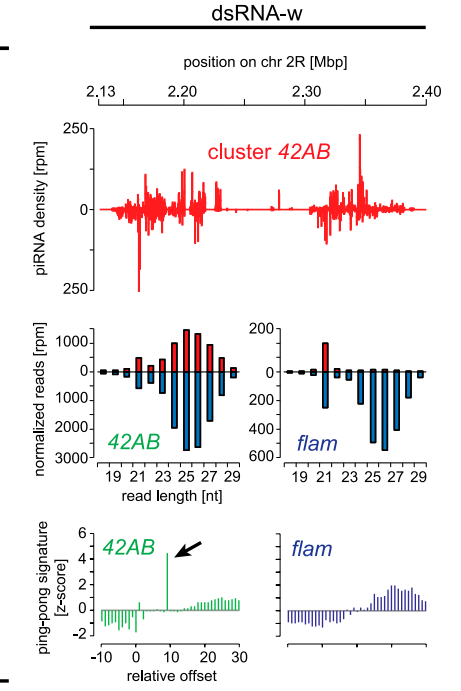

C

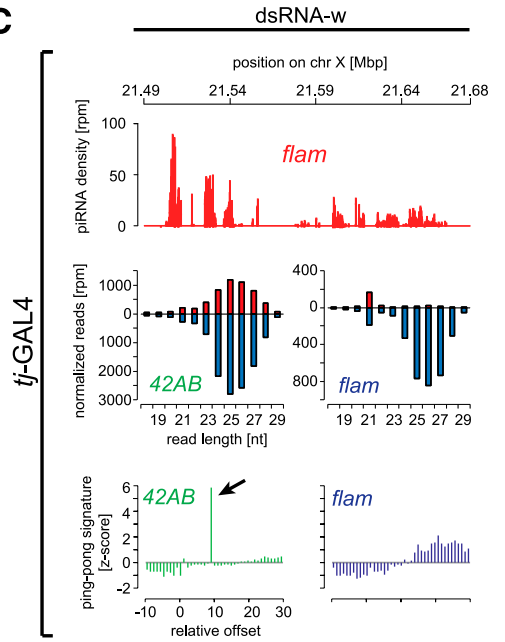

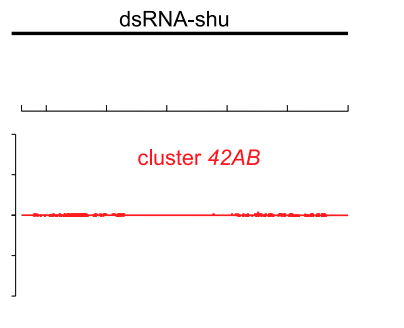
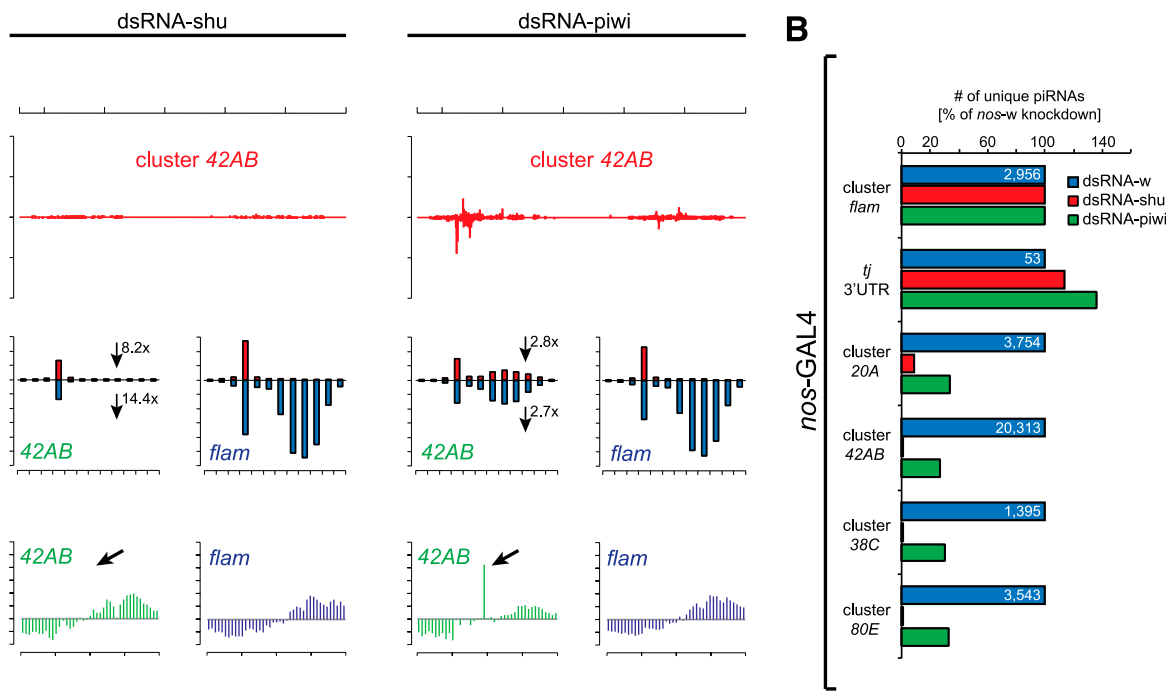

dsRNA-piwi
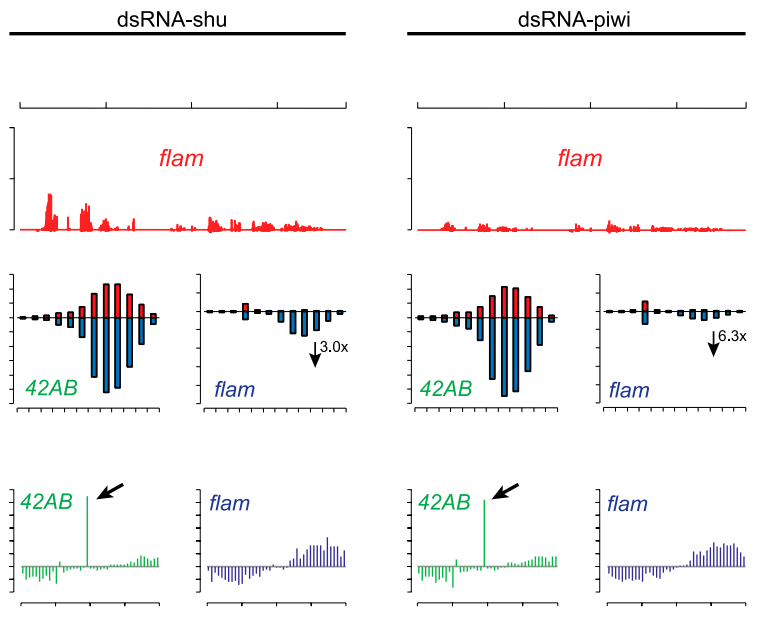
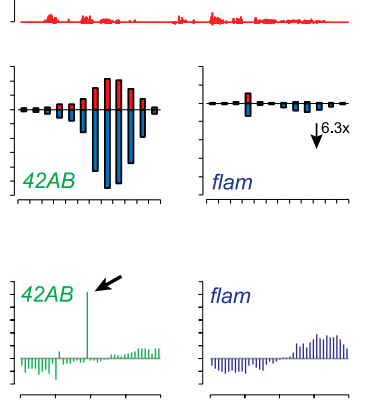

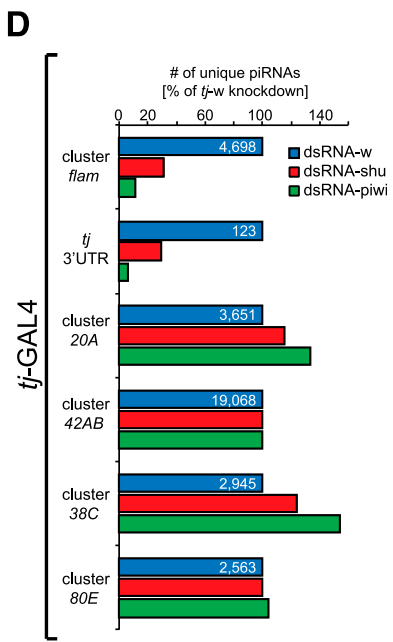

FIGURE 3. Knockdown of shu causes loss of cluster-derived piRNAs in both somatic and germline tissues. (A) At the top is shown a histogram of small RNAs mapping to the germline-specific $42 A B$ cluster in flies expressing the indicated dsRNAs specifically in germ cells. In the middle, the size distribution of RNAs derived from each strand of the $42 A B$ and flamenco clusters is shown as a histogram. At the bottom are histograms reflecting the relative enrichment of RNAs overlapping by the indicated number of nucleotides, plotted by $Z$-score, for the $42 A B$ and flamenco clusters in the indicated knockdown animals. The peak at position 9 (arrow) is indicative of a ping-pong interaction. (B) A histogram shows relative piRNA levels for a series of germline and somatic clusters. Total reads were normalized across libraries to piRNAs mapping to flamenco, which is unaffected in germline-specific knockdowns. For each cluster, changes in mapping piRNAs are shown with reference to the white control, which is set to $100 \%$. $C$ and $D$ are similar to $A$ and $B$ except that dsRNA expression is driven by a follicle cell-specific tj-GAL4 driver. In $C$, at the top, reads are shown mapping to the soma-specific flamenco cluster. In $D$, reads are normalized across libraries to those derived from $42 A B$, whose activity is not affected in the soma-specific knockdown.

(Brennecke et al. 2007), with loading of Aub and Ago3 occurring independently of Piwi function. Small RNAs mapping to this cluster in the shu-depleted germline also showed a clear reduction in ping-pong signatures, defined as the frequency of reads with a paired opposite strand read overlapping by $10 \mathrm{nt}$ (Fig. 3A). In contrast, piwi, which does not participate significantly in ping-pong amplification, had no effect on ping-ping signatures upon knockdown. The effects of $s h u$ knockdown appear to be specific to the piRNA pathway. Reads corresponding to miRNAs were not reduced in $s h u$ knockdown animals (Supplemen- tal Fig. S3). Though enforced Dicer-2 expression generally increased the endo-siRNA fraction, we did not note any further effect of $s h u$ knockdown, even on endo-siRNAs mapping to piRNA clusters (e.g., 42AB) (Fig. 3A).

We also analyzed effects of shu or piwi depletion on other piRNA clusters. We compared reads that could be uniquely mapped to each annotated cluster to the white knockdown controls. Reads were set to $100 \%$ in the white library (normalized read number for the white knockdown library is show as a blue bar). piRNAs derived from the 3' UTR of traffic jam, a genic locus that produces piRNAs only in 
follicle cells, showed no impact of shu and piwi knockdowns (Fig. 3B), as expected. In contrast, all germline clusters analyzed showed a dramatic reduction of piRNA levels upon expression of nos-GAL4-driven shu dsRNAs $(<10 \%$ remaining as compared to white RNAi). Depletion of Piwi had similar effects, although the reduction was less profound ( $\sim 30 \%$ of white levels, as seen for $42 A B)$, probably due to intact Ago3 and Aub loading.

Primary and secondary piRNA biogenesis mechanisms in the germline exhibit some degree of interdependence. For example, disruption of ping-pong in ago3 mutants or upon Aub knockdown feeds back and reduces the number of primary piRNAs loading into Piwi through unknown mechanisms (Li et al. 2009; Wang and Elgin 2011). Follicle cells, which are of somatic origin, express no detectable Aub or Ago 3 and do not use ping-pong amplification. Thus, we directly tested the involvement of $s h u$ in primary piRNA production by sequencing small RNAs from tj-GAL4driven dsRNA in ovaries. PiRNA-sized small RNAs were normalized using the number of unique reads mapping to the germline-specific $42 A B$ locus, which is unaffected by tj-GAL4-mediated knockdowns.

The sole somatic, unidirectional flamenco cluster produces abundant piRNAs that load only the Piwi protein. Thus, as expected, depletion of piwi caused a significant reduction in piRNAs derived from this locus (5.2-fold) (Fig. 3C,D). Follicular knockdown of shu also produced a marked reduction in flam piRNAs (2.9-fold) (Fig. 3C,D). As expected, piRNAs uniquely mapped to flam showed no ping-pong signature in any of the somatic knockdowns. Reads corresponding to germline clusters remained unchanged in piRNA abundance, with no shift in size profiles or ping-pong signatures, indicating that, as expected, the pathway remains fully functional in germ cells of animals that have lost shu expression only in the soma. As in germ cell-specific knockdowns, miRNA abundance was unaffected (Supplemental Fig. S3).

Mapping small RNAs from our germline-specific knockdown animals to a set of known Drosophila transposon consensus sequence further supported a general requirement for $s h u$ in piRNA accumulation. We retained in our analysis only the 75 transposons with the highest abundance of corresponding piRNAs. Previous reports have demonstrated substantial expression biases for many transposons, with some showing preferential expression in the somatic lineage and others being found predominantly in germline lineages (Malone et al. 2009). For the set of germline-enriched transposons, nos-GAL4-driven dsRNAshu substantially affected all known elements, reducing overall piRNA levels (Fig. 4A). In general, sense and antisense piRNAs were depleted to roughly similar extents, suggesting that loading of all three PIWI clade proteins is affected by loss of shu. In contrast, only a subset of transposons showed depletion of piRNAs in the nos-GAL4-driven piwi knockdowns. Elements with a known somatic expres- sion bias, including ZAM, tabor, gypsy, and others (indicated by red dots), show little or no reduction in piRNA levels upon germline knockdown of either shu or piwi (Fig. 4A). Transposable elements with strong germline signatures (green asterisks), like the LINE element Rt $1 b$ or the LTR transposon roo (pao family), not only showed a severe reduction of their corresponding piRNA levels but also demonstrated a dramatic loss of ping-pong signatures (Fig. 4B). In contrast, soma-specific elements retain their piRNA levels and generally lack ping-pong signatures. As an example of such an element, piRNA levels for the LTR element ZAM (gypsy family) are shown (Fig. 4B, bottom).

\section{Summary}

A combination of biochemical and genetic approaches are beginning to link a substantial number of proteins to functions in the piRNA pathway. Some act exclusively in primary piRNA biogenesis and affect small RNAs in both the germline and somatic compartments of the Drosophila ovary (Malone et al. 2009; Haase et al. 2010; Olivieri et al. 2010; Saito et al. 2010; Handler et al. 2011; Zamparini et al. 2011). Others function exclusively in the germline, and these tend to selectively affect the ping-pong cycle that hones piRNA populations in response to the expression of transposon mRNAs or factors implicated in germline cluster transcription (Klattenhoff et al. 2009; Li et al. 2009; Patil and Kai 2010; Pane et al. 2011; Zhang et al. 2011; Anand and Kai 2012). Here, we followed clues initially provided by proteomic analysis of Piwi-family protein complexes in mice to link shutdown, a gene previously shown to be required for fertility in Drosophila females (Schupbach and Wieschaus 1991; Munn and Steward 2000), to the piRNA pathway.

Analysis of transposon expression patterns and small RNA libraries in shu knockdown cells and animals suggests a role either in piRNA biogenesis or in piRNA stabilization, perhaps by fostering loading of piRNAs into PIWI-family proteins. Shutdown is a member of the FKBP family and its constituent domains have been ascribed PPIase activity and the ability to interact with the HSP90 family of chaperone proteins. Either of these activities could underlie the role of Shutdown in the piRNA pathway. In particular, studies of the Argonaute clade have implicated HSP family chaperones as critical cofactors for small RNA loading (Iki et al. 2010; Iwasaki et al. 2010; Miyoshi et al. 2010; Iki et al. 2011). However, evolutionary comparisons indicate that both the PPI and HSP90binding domains harbor variations that reduce activity when introduced into other well-studied FKBP family members. Thus, understanding the true role of Shutdown in both primary biogenesis and the ping-pong cycle will await further genetic analysis and the development of biochemical systems that recapitulate aspects of the piRNA pathway in vitro. 


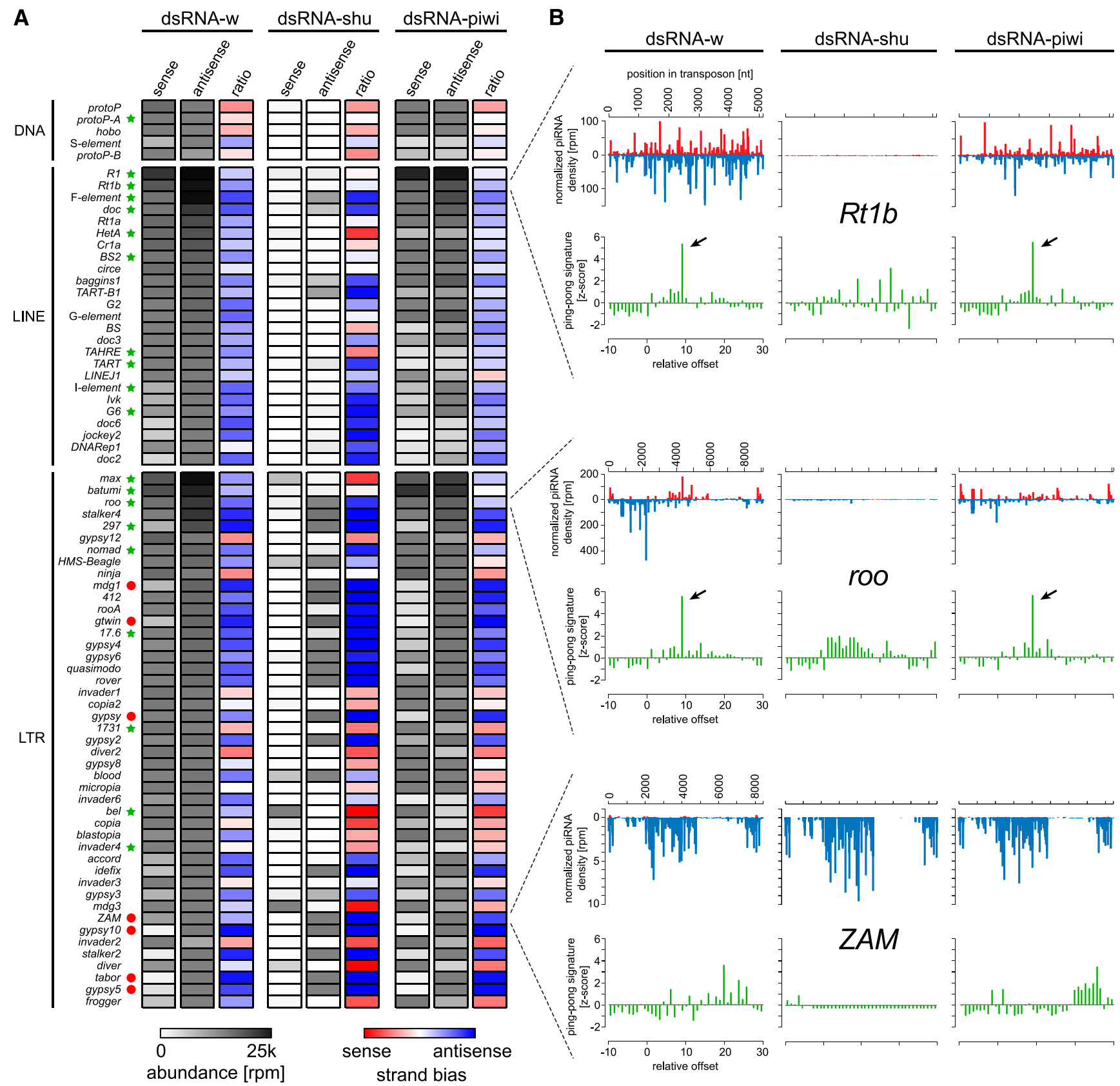

FIGURE 4. Loss of transposon control in shu knockdowns is a consequence of piRNA loss. (A) The heat map displays changes in piRNA abundance for each germline knockdown (as indicated) for the 75 elements most heavily targeted in our strain. Sense and antisense, with respect to the transposon coding strand, are quantified separately (gray heat maps), and their ratio is also indicated (red-blue heat map). ( $B$ ) For three transposons, piRNAs are plotted along the length of the consensus sequence (upper) and a histogram of overlap between sense and antisense species (lower) is presented to indicate the degree of ping-pong (arrow highlights peak at position 9). Data are presented for shu and piwi knockdown and for a control (white). Two transposons with strong expression in the germline, Rt $1 b$ and roo (top and middle), are shown in comparison to a somatically biased element, ZAM. Since knockdown is germline specific, ZAM piRNAs are unaffected.

\section{MATERIALS AND METHODS}

\section{Fly stocks and handling}

Drosophila UAS-dsRNA strains were obtained from the VDRC. nosGAL4 and tj-GAL4 driver lines were obtained from Bloomington and Kyoto, respectively (see Supplemental Table S1). For knock- down experiments, five males from dsRNA stocks were crossed with five virgin females expressing the desired GAL4 driver. Fertility of the FKBP-family F1 knockdown females was estimated by counting the number of eggs laid and crawling larvae $7 \mathrm{~d}$ post transfer to fresh media. Quantitative fertility measurements (shown in Fig. 1D) were obtained by transferring 3-d-old male and female F1 offspring (10 each) to grape-agar plates for 
$4 \mathrm{~h}$ and counting the eggs laid. Hatching frequencies were ascertained after $24 \mathrm{~h}$ (measurements were carried out in triplicate). For qPCR, small RNA libraries, and immunofluorescence experiments, ovaries were dissected from 2- to 3-d-old females fed with fresh yeast paste.

\section{Expression of tagged transgenes in OSS}

Full-length coding sequences of Shu, Piwi, Armi-RB, and Zuc were amplified from Drosophila ovary cDNA, cloned into pENTR/ $\mathrm{D}-\mathrm{TOPO}$, and recombined into $\mathrm{N}$ - or C-terminal GFP destination vectors of the Drosophila Gateway collection (Terence Murphy, Carnegie Institute of Washington, Baltimore, MD). Shu was cloned into pAGW and pAWG, Zuc into pUWG, and Piwi and Armi into pUGW. Cells were transfected using Xfect reagent (Clontech) and costained with DAPI and MitoTracker Red CMXRos (Invitrogen).

\section{RNA isolation, reverse transcription, $q P C R$}

Ovaries were dissected into cold $1 \times$ PBS. Total RNA was extracted using TRIzol reagent (Invitrogen) following the manufacturer's instructions. One microgram RNA was treated with DNase I Amplification Grade (Invitrogen) according to the manufacturer's instructions. Complementary DNA was prepared by reverse transcription using oligo $(\mathrm{dT})_{20}$ primer and SuperScript III Reverse Transcriptase (Invitrogen). qPCR was carried out using SYBR Green PCR Master Mix (Applied Biosystems) and primers listed in Supplemental Table S2 on a Chromo4 Real-Time PCR Detector (BioRad). Transcripts were quantitated by the $\Delta \Delta \mathrm{C}_{\mathrm{t}}$ method (Livak and Schmittgen 2001), and normalized to transcript levels of $r p 49$. Fold changes are expressed relative to control dsRNA-white knockdown RNA. Significance was calculated using a one-tailed heteroscedastic Student $t$-test of rp49-subtracted transposon $\mathrm{c}(\mathrm{t})$ values. All experiments were carried out in triplicates, with the average results shown.

\section{Immunofluorescence}

Ovaries were fixed in freshly prepared $4 \%$ paraformaldehyde for $20 \mathrm{~min}$ at room temperature. Blocking and permeabilization were carried out simultaneously in wash buffer $(50 \mathrm{mM}$ Tris at $\mathrm{pH} 6.8$, $150 \mathrm{mM} \mathrm{NaCl}, 0.5 \% \mathrm{NP}-40$ ) supplemented with bovine serum albumin $(5 \mathrm{mg} / \mathrm{mL})$. All primary antibodies were diluted 1:1000 and incubated overnight at $4^{\circ} \mathrm{C}$ in wash buffer plus $1 \mathrm{mg} / \mathrm{mL}$ BSA. Anti-Ago3 and Anti-Piwi were generated in our laboratory (Brennecke et al. 2007); monoclonal mouse anti-Aub was provided by Mikiko Siomi (Nishida et al. 2007); and rabbit anti-Vasa (d-260) was purchased from Santa Cruz. Secondary AlexaFluor-488 and -568 antibodies were purchased from Invitrogen and used at 1:1000. Images were acquired on a Perkin Elmer UltraVIEW spinning disk confocal microscope.

\section{RNAseq data analysis}

For transcriptome libraries, $1 \mu \mathrm{g}$ of total RNA from OSS cells transfected with GFP control dsRNA was used as input for the Illumina mRNA-Seq sample prep kit (catalog no. RS-930-1001). Libraries were made following the instructions by the manufacturer and sequenced on the Illumina GAII platform. RNAseq data were deposited to the Gene Expression Omnibus database (www. ncbi.nlm.nih.gov/geo/) under accession no. GSE38090. Publically available ovarian RNAseq data (GEO accession no. GSM424751) (Gan et al. 2010) were reanalyzed for this study. Raw sequence reads were iteratively mapped to the Drosophila genome (version dm3) using Bowtie (Langmead et al. 2009) with a tolerance of up to two mismatches. Remaining reads were also mapped to RefGene-annotated exon junctions with TopHat (Trapnell et al. 2009). Transcripts were quantitated using Cufflinks (Trapnell et al. 2010) and expressed as fragments per kilobase per million reads (fkpm) for relative comparison of FKBP family mRNA expression in the ovary.

\section{Small RNA libraries and bioinformatic analysis}

Small RNAs were cloned as described (Brennecke et al. 2007). For this study, the following small RNA libraries from total RNAs were prepared:

19-28 nt from $t$-GAL4-driven dsRNA against white, 19-28 nt from $t j$-GAL4-driven dsRNA against shu, 19-28 nt from tj-GAL4-driven dsRNA against piwi, 19-28 nt from nos-GAL4-driven dsRNA against white, 19-28 nt from nos-GAL4-driven dsRNA against shu, and 19-28 nt from nos-GAL4-driven dsRNA against piwi.

Libraries were sequenced in-house using the Illumina GAII sequencing platform. Small RNA sequences were deposited in the Gene Expression Omnibus database (www.ncbi.nlm.nih.gov/geo/) under accession no. GSE38089. The analysis of small RNA libraries was performed similarly as described (Czech et al. 2008). In brief, Illumina reads were stripped of the $3^{\prime}$ linker and collapsed, and the resulting small RNA sequences were matched to the Drosophila release 5 genome (version $\mathrm{dm} 3$ ) without mismatches. Only reads that met these conditions were subjected to further analyses. For annotations we used a combination of UCSC (repeats/transposons; noncoding RNAs), miRBase (microRNAs), and FlyBase (protein coding genes; noncoding RNAs) tracks, as well as custom tracks (for synthetic markers, endo-siRNAs from structured loci, and $\mathrm{miR}$ and $\mathrm{miR}^{\star}$ strands) with different priorities. For comparison of small RNA counts between samples, libraries of dsRNAwhite samples were set to 1 million reads. Next, all libraries were normalized based on unique piRNA-size mappers to the flamenco (for nos-GAL4 knockdowns) or $42 A B$ (for tj-GAL4 knockdowns) piRNA clusters. Heat maps were created by plotting the abundance of sequences (all piRNAs to a given transposable element or individual miRNA strands) as well as their strand bias within the indicated libraries.

\section{Ping-pong analysis}

For each piRNA, the relative frequency ( $Z$-score) of an existing "neighbor" piRNA on the opposite strand within a certain window (10-nt upstream of and 30-nt downstream from each 5' end of a piRNA) was calculated. In the case of germline and somatic piRNA clusters, this analysis was based on genomic mapping coordinates. For transposons, the $5^{\prime}$ coordinate of each mapping event to the respective transposon consensus sequence was used. Calculated frequencies were based on total cloning count. A spike at position 9 indicates more than average partners with a $10-\mathrm{nt}$ overlap and is a signature of ping-pong amplified piRNAs. 


\section{DATA DEPOSITION}

RNAseq data and small RNA sequences were deposited in the Gene Expression Omnibus database (www.ncbi.nlm.nih.gov/geo/) under accession no. GSE38098.

\section{SUPPLEMENTAL MATERIAL}

Supplemental material is available for this article.

\section{ACKNOWLEDGMENTS}

We thank members of the Hannon laboratory for helpful discussion, Yang Yu for providing DNA constructs, and Assaf Gordon for help with ping-pong analysis. We thank the VDRC, Kyoto, and Bloomington stock centers for fly stocks, and Julius Brennecke (IMBA, Austria) and Mikiko Siomi (Keio University, Japan) for antibodies. J.P. is supported by the American Cancer Society (award no. 121614-PF-11-277-01-RMC). B.C. was supported by the Boehringer Ingelheim Fonds. P.M.G. is supported by the NIH (grant 5T32GM065094) and by a William Randolph Hearst Foundation Scholarship. Work in the Hannon laboratory is supported by grants from the NIH and by a kind gift from Kathryn W. Davis. G.J.H. is an investigator of the HHMI.

Received May 14, 2012; accepted May 15, 2012.

\section{REFERENCES}

Ahearn IM, Tsai FD, Court H, Zhou M, Jennings BC, Ahmed M, Fehrenbacher N, Linder ME, Philips MR. 2011. FKBP12 binds to acylated H-ras and promotes depalmitoylation. Mol Cell 41: 173185.

Allan RK, Ratajczak T. 2011. Versatile TPR domains accommodate different modes of target protein recognition and function. Cell Stress Chaperones 16: 353-367.

Anand A, Kai T. 2012. The tudor domain protein kumo is required to assemble the nuage and to generate germline piRNAs in Drosophila. EMBO J 31: 870-882.

Aravin AA, Naumova NM, Tulin AV, Vagin VV, Rozovsky YM, Gvozdev VA. 2001. Double-stranded RNA-mediated silencing of genomic tandem repeats and transposable elements in the D. melanogaster germline. Curr Biol 11: 1017-1027.

Aravin A, Gaidatzis D, Pfeffer S, Lagos-Quintana M, Landgraf P, Iovino N, Morris P, Brownstein MJ, Kuramochi-Miyagawa S, Nakano T, et al. 2006. A novel class of small RNAs bind to MILI protein in mouse testes. Nature 442: 203-207.

Bozzetti MP, Massari S, Finelli P, Meggio F, Pinna LA, Boldyreff B, Issinger OG, Palumbo G, Ciriaco C, Bonaccorsi S, et al. 1995. The Ste locus, a component of the parasitic cry-Ste system of Drosophila melanogaster, encodes a protein that forms crystals in primary spermatocytes and mimics properties of the $\beta$ subunit of casein kinase 2. Proc Natl Acad Sci 92: 6067-6071.

Brennecke J, Aravin AA, Stark A, Dus M, Kellis M, Sachidanandam R, Hannon GJ. 2007. Discrete small RNA-generating loci as master regulators of transposon activity in Drosophila. Cell 128: 1089-1103.

Chalvet F, Teysset L, Terzian C, Prud'homme N, Santamaria P, Bucheton A, Pelisson A. 1999. Proviral amplification of the Gypsy endogenous retrovirus of Drosophila melanogaster involves envindependent invasion of the female germline. EMBO J 18: 26592669.

Chambeyron S, Popkova A, Payen-Groschene G, Brun C, Laouini D, Pelisson A, Bucheton A. 2008. piRNA-mediated nuclear accumu- lation of retrotransposon transcripts in the Drosophila female germline. Proc Natl Acad Sci 105: 14964-14969.

Chen Y, Pane A, Schupbach T. 2007. Cutoff and aubergine mutations result in retrotransposon upregulation and checkpoint activation in Drosophila. Curr Biol 17: 637-642.

Chintapalli VR, Wang J, Dow JA. 2007. Using FlyAtlas to identify better Drosophila melanogaster models of human disease. Nat Genet 39: 715-720.

Cook HA, Koppetsch BS, Wu J, Theurkauf WE. 2004. The Drosophila SDE3 homolog armitage is required for oskar mRNA silencing and embryonic axis specification. Cell 116: 817-829.

Cox DN, Chao A, Lin H. 2000. piwi encodes a nucleoplasmic factor whose activity modulates the number and division rate of germline stem cells. Development 127: 503-514.

Crackower MA, Kolas NK, Noguchi J, Sarao R, Kikuchi K, Kaneko H, Kobayashi E, Kawai Y, Kozieradzki I, Landers R, et al. 2003. Essential role of Fkbp6 in male fertility and homologous chromosome pairing in meiosis. Science 300: 1291-1295.

Czech B, Malone CD, Zhou R, Stark A, Schlingeheyde C, Dus M, Perrimon N, Kellis M, Wohlschlegel JA, Sachidanandam R, et al. 2008. An endogenous small interfering RNA pathway in Drosophila. Nature 453: 798-802.

DeCenzo MT, Park ST, Jarrett BP, Aldape RA, Futer O, Murcko MA, Livingston DJ. 1996. FK506-binding protein mutational analysis: defining the active-site residue contributions to catalysis and the stability of ligand complexes. Protein Eng 9: 173-180.

Feschotte C. 2008. Transposable elements and the evolution of regulatory networks. Nat Rev Genet 9: 397-405.

Gan Q, Chepelev I, Wei G, Tarayrah L, Cui K, Zhao K, Chen X. 2010. Dynamic regulation of alternative splicing and chromatin structure in Drosophila gonads revealed by RNA-seq. Cell Res 20: 763-783.

Girard A, Sachidanandam R, Hannon GJ, Carmell MA. 2006. A germline-specific class of small RNAs binds mammalian Piwi proteins. Nature 442: 199-202.

Gollan PJ, Bhave M. 2010. Genome-wide analysis of genes encoding FK506-binding proteins in rice. Plant Mol Biol 72: 1-16.

Gonzalez-Reyes A, Elliott H, St Johnston D. 1997. Oocyte determination and the origin of polarity in Drosophila: the role of the spindle genes. Development 124: 4927-4937.

Grimson A, Srivastava M, Fahey B, Woodcroft BJ, Chiang HR, King N, Degnan BM, Rokhsar DS, Bartel DP. 2008. Early origins and evolution of microRNAs and Piwi-interacting RNAs in animals. Nature 455: 1193-1197.

Gunawardane LS, Saito K, Nishida KM, Miyoshi K, Kawamura Y, Nagami T, Siomi H, Siomi MC. 2007. A slicer-mediated mechanism for repeat-associated siRNA $5^{\prime}$ end formation in Drosophila. Science 315: 1587-1590.

Haase AD, Fenoglio S, Muerdter F, Guzzardo PM, Czech B, Pappin DJ, Chen C, Gordon A, Hannon GJ. 2010. Probing the initiation and effector phases of the somatic piRNA pathway in Drosophila. Genes Dev 24: 2499-2504.

Handler D, Olivieri D, Novatchkova M, Gruber FS, Meixner K, Mechtler K, Stark A, Sachidanandam R, Brennecke J. 2011. A systematic analysis of Drosophila TUDOR domain-containing proteins identifies Vreteno and the Tdrd12 family as essential primary piRNA pathway factors. EMBO J 30: 3977-3993.

Houwing S, Kamminga LM, Berezikov E, Cronembold D, Girard A, van den Elst H, Filippov DV, Blaser H, Raz E, Moens CB, et al. 2007. A role for Piwi and piRNAs in germ cell maintenance and transposon silencing in Zebrafish. Cell 129: 69-82.

Iki T, Yoshikawa M, Nishikiori M, Jaudal MC, Matsumoto-Yokoyama E, Mitsuhara I, Meshi T, Ishikawa M. 2010. In vitro assembly of plant RNA-induced silencing complexes facilitated by molecular chaperone HSP90. Mol Cell 39: 282-291.

Iki T, Yoshikawa M, Meshi T, Ishikawa M. 2011. Cyclophilin 40 facilitates HSP90-mediated RISC assembly in plants. EMBO J 31: 267-278.

Iwasaki S, Kobayashi M, Yoda M, Sakaguchi Y, Katsuma S, Suzuki T, Tomari Y. 2010. Hsc70/Hsp90 chaperone machinery mediates 
ATP-dependent RISC loading of small RNA duplexes. Mol Cell 39: 292-299.

Kamphausen T, Fanghanel J, Neumann D, Schulz B, Rahfeld JU. 2002. Characterization of Arabidopsis thaliana AtFKBP42 that is membrane-bound and interacts with Hsp90. Plant J 32: 263 276.

Kang CB, Hong Y, Dhe-Paganon S, Yoon HS. 2008. FKBP family proteins: immunophilins with versatile biological functions. Neurosignals 16: 318-325.

Khurana JS, Theurkauf W. 2010. piRNAs, transposon silencing, and Drosophila germline development. J Cell Biol 191: 905-913.

Kim A, Terzian C, Santamaria P, Pelisson A, Purd'homme N, Bucheton A. 1994. Retroviruses in invertebrates: the gypsy retrotransposon is apparently an infectious retrovirus of Drosophila melanogaster. Proc Natl Acad Sci 91: 1285-1289.

Klattenhoff C, Bratu DP, McGinnis-Schultz N, Koppetsch BS, Cook HA, Theurkauf WE. 2007. Drosophila rasiRNA pathway mutations disrupt embryonic axis specification through activation of an ATR/Chk2 DNA damage response. Dev Cell 12: 4555.

Klattenhoff C, Xi H, Li C, Lee S, Xu J, Khurana JS, Zhang F, Schultz N, Koppetsch BS, Nowosielska A, et al. 2009. The Drosophila HP1 homolog Rhino is required for transposon silencing and piRNA production by dual-strand clusters. Cell 138: 11371149.

Langmead B, Trapnell C, Pop M, Salzberg SL. 2009. Ultrafast and memory-efficient alignment of short DNA sequences to the human genome. Genome Biol 10: R25. doi: 10.1186/gb-2009-10-3-r25.

Lau NC, Seto AG, Kim J, Kuramochi-Miyagawa S, Nakano T, Bartel DP, Kingston RE. 2006. Characterization of the piRNA complex from rat testes. Science 313: 363-367.

Li C, Vagin VV, Lee S, Xu J, Ma S, Xi H, Seitz H, Horwich MD, Syrzycka M, Honda BM, et al. 2009. Collapse of germline piRNAs in the absence of Argonaute 3 reveals somatic piRNAs in flies. Cell 137: 509-521.

Li L, Lou Z, Wang L. 2011. The role of FKBP5 in cancer aetiology and chemoresistance. Br J Cancer 104: 19-23.

Lim AK, Kai T. 2007. Unique germ-line organelle, nuage, functions to repress selfish genetic elements in Drosophila melanogaster. Proc Natl Acad Sci 104: 6714-6719.

Lin H, Spradling AC. 1997. A novel group of pumilio mutations affects the asymmetric division of germline stem cells in the Drosophila ovary. Development 124: 2463-2476.

Livak KJ, Schmittgen TD. 2001. Analysis of relative gene expression data using real-time quantitative PCR and the $2^{-\Delta \Delta C}$ method. Methods 25: 402-408.

Malone CD, Hannon GJ. 2009. Small RNAs as guardians of the genome. Cell 136: 656-668.

Malone CD, Brennecke J, Dus M, Stark A, McCombie WR, Sachidanandam R, Hannon GJ. 2009. Specialized piRNA pathways act in germline and somatic tissues of the Drosophila ovary. Cell 137: $522-535$.

McClintock B. 1953. Induction of instability at selected loci in maize. Genetics 38: 579-599.

Miyoshi T, Takeuchi A, Siomi H, Siomi MC. 2010. A direct role for Hsp90 in pre-RISC formation in Drosophila. Nat Struct Mol Biol 17: $1024-1026$.

Munn K, Steward R. 2000. The shut-down gene of Drosophila melanogaster encodes a novel FK506-binding protein essential for the formation of germline cysts during oogenesis. Genetics 156: $245-256$.

Nishida KM, Saito K, Mori T, Kawamura Y, Nagami-Okada T, Inagaki S, Siomi H, Siomi MC. 2007. Gene silencing mechanisms mediated by Aubergine-piRNA complexes in Drosophila male gonad. RNA 13: 1911-1922.

Olivieri D, Sykora MM, Sachidanandam R, Mechtler K, Brennecke J. 2010. An in vivo RNAi assay identifies major genetic and cellular requirements for primary piRNA biogenesis in Drosophila. EMBO J 29: 3301-3317.
Orsi GA, Joyce EF, Couble P, McKim KS, Loppin B. 2010. Drosophila $I-R$ hybrid dysgenesis is associated with catastrophic meiosis and abnormal zygote formation. J Cell Sci 123: 3515-3524.

Pane A, Wehr K, Schupbach T. 2007. zucchini and squash encode two putative nucleases required for rasiRNA production in the Drosophila germline. Dev Cell 12: 851-862.

Pane A, Jiang P, Zhao DY, Singh M, Schupbach T. 2011. The Cutoff protein regulates piRNA cluster expression and piRNA production in the Drosophila germline. EMBO J 30: 4601-4615.

Patil VS, Kai T. 2010. Repression of retroelements in Drosophila germline via piRNA pathway by the Tudor domain protein Tejas. Current biology: CB 20: 724-730.

Pelisson A, Song SU, Prud'homme N, Smith PA, Bucheton A, Corces VG. 1994. Gypsy transposition correlates with the production of a retroviral envelope-like protein under the tissuespecific control of the Drosophila flamenco gene. EMBO J 13: $4401-4411$.

Pratt WB. 1998. The hsp90-based chaperone system: involvement in signal transduction from a variety of hormone and growth factor receptors. Proc Soc Exp Biol Med 217: 420-434.

Pratt WB, Galigniana MD, Harrell JM, DeFranco DB. 2004. Role of hsp90 and the hsp90-binding immunophilins in signalling protein movement. Cell Signal 16: 857-872.

Ratajczak T, Carrello A. 1996. Cyclophilin 40 (CyP-40), mapping of its hsp90 binding domain and evidence that FKBP52 competes with CyP-40 for hsp90 binding. J Biol Chem 271: 29612965.

Saito K, Nishida KM, Mori T, Kawamura Y, Miyoshi K, Nagami T, Siomi H, Siomi MC. 2006. Specific association of Piwi with rasiRNAs derived from retrotransposon and heterochromatic regions in the Drosophila genome. Genes Dev 20: 2214-2222.

Saito K, Ishizu H, Komai M, Kotani H, Kawamura Y, Nishida KM, Siomi H, Siomi MC. 2010. Roles for the Yb body components Armitage and $\mathrm{Yb}$ in primary piRNA biogenesis in Drosophila. Genes Dev 24: 2493-2498.

Schupbach T, Wieschaus E. 1989. Female sterile mutations on the second chromosome of Drosophila melanogaster. I. Maternal effect mutations. Genetics 121: 101-117.

Schupbach T, Wieschaus E. 1991. Female sterile mutations on the second chromosome of Drosophila melanogaster. II. Mutations blocking oogenesis or altering egg morphology. Genetics 129: 1119-1136.

Senti KA, Brennecke J. 2010. The piRNA pathway: a fly's perspective on the guardian of the genome. Trends Genet 26: 499-509.

Smith MR, Willmann MR, Wu G, Berardini TZ, Moller B, Weijers D, Poethig RS. 2009. Cyclophilin 40 is required for microRNA activity in Arabidopsis. Proc Natl Acad Sci 106: 5424-5429.

Szakmary A, Reedy M, Qi H, Lin H. 2009. The Yb protein defines a novel organelle and regulates male germline stem cell selfrenewal in Drosophila melanogaster. J Cell Biol 185: 613-627.

Theurkauf WE, Klattenhoff C, Bratu DP, McGinnis-Schultz N, Koppetsch BS, Cook HA. 2006. rasiRNAs, DNA damage, and embryonic axis specification. Cold Spring Harb Symp Quant Biol 71: 171-180.

Trapnell C, Pachter L, Salzberg SL. 2009. TopHat: discovering splice junctions with RNA-Seq. Bioinformatics 25: 1105-1111.

Trapnell C, Williams BA, Pertea G, Mortazavi A, Kwan G, van Baren MJ, Salzberg SL, Wold BJ, Pachter L. 2010. Transcript assembly and quantification by RNA-Seq reveals unannotated transcripts and isoform switching during cell differentiation. Nat Biotechnol 28: $511-515$.

Vagin VV, Sigova A, Li C, Seitz H, Gvozdev V, Zamore PD. 2006. A distinct small RNA pathway silences selfish genetic elements in the germline. Science 313: 320-324.

Vagin VV, Wohlschlegel J, Qu J, Jonsson Z, Huang X, Chuma S, Girard A, Sachidanandam R, Hannon GJ, Aravin AA. 2009. Proteomic analysis of murine Piwi proteins reveals a role for arginine methylation in specifying interaction with Tudor family members. Genes Dev 23: 1749-1762. 
Van Duyne GD, Standaert RF, Karplus PA, Schreiber SL, Clardy J. 1993. Atomic structures of the human immunophilin FKBP-12 complexes with FK506 and rapamycin. J Mol Biol 229: 105-124.

Wang SH, Elgin SC. 2011. Drosophila Piwi functions downstream of piRNA production mediating a chromatin-based transposon silencing mechanism in female germ line. Proc Natl Acad Sci 108: 21164-21169.

Ward BK, Allan RK, Mok D, Temple SE, Taylor P, Dornan J, Mark PJ, Shaw DJ, Kumar P, Walkinshaw MD, et al. 2002. A structurebased mutational analysis of cyclophilin 40 identifies key residues in the core tetratricopeptide repeat domain that mediate binding to Hsp90. J Biol Chem 277: 40799-40809.
Wilson JE, Connell JE, Macdonald PM. 1996. aubergine enhances oskar translation in the Drosophila ovary. Development 122: 16311639.

Zamparini AL, Davis MY, Malone CD, Vieira E, Zavadil J, Sachidanandam R, Hannon GJ, Lehmann R. 2011. Vreteno, a gonad-specific protein, is essential for germline development and primary piRNA biogenesis in Drosophila. Development 138: 4039-4050.

Zhang Z, Xu J, Koppetsch BS, Wang J, Tipping C, Ma S, Weng Z, Theurkauf WE, Zamore PD. 2011. Heterotypic piRNA Ping-Pong requires qin, a protein with both E3 ligase and Tudor domains. Mol Cell 44: 572-584. 

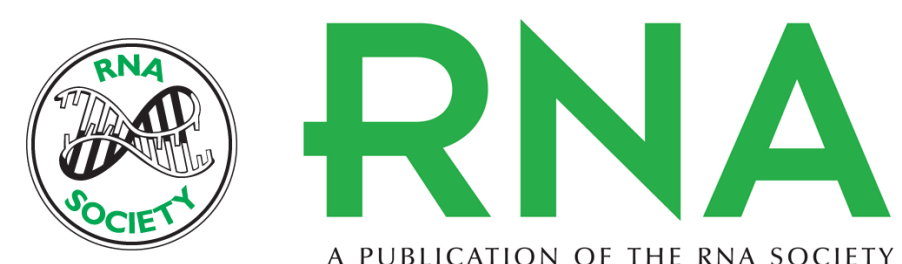

A PUBLICATION OF THE RNA SOCIETY

\section{shutdown is a component of the Drosophila piRNA biogenesis machinery}

Jonathan B. Preall, Benjamin Czech, Paloma M. Guzzardo, et al.

RNA 2012 18: 1446-1457 originally published online July 2, 2012

Access the most recent version at doi:10.1261/rna.034405.112

\section{Supplemental http://rnajournal.cshlp.org/content/suppl/2012/06/05/rna.034405.112.DC1 \\ Material}

References This article cites 75 articles, 26 of which can be accessed free at: http://rnajournal.cshlp.org/content/18/8/1446.full.html\#ref-list-1

Open Access Freely available online through the RNA Open Access option.

License Freely available online through the RNA Open Access option.

Email Alerting Receive free email alerts when new articles cite this article - sign up in the box at the Service top right corner of the article or click here. 